\title{
Thymectomy in Myasthenia Gravis
}

\section{(ㄷ) (i) ()ㅇㅇ $\Theta$}

Authors

Jens C. Rückert ${ }^{1}$, Marc Swierzy ${ }^{1}$, Siegfried Kohler ${ }^{2}$, Andreas Meisel ${ }^{2}$, Mahmoud Ismail ${ }^{1}$

Affiliation

1 Kompetenzzentrum für Thoraxchirurgie (CCM,CVK,CBF), Chirurgische Klinik Campus Charité Mitte / Campus Virchow-Klinikum; Charité - Universitätsmedizin Berlin

2 Integriertes Myasthenie Zentrum, Klinik für Neurologie, NeuroCure Clinical Research Center, Charité Universitätsmedizin Berlin

Key words

Thymectomy, robotic, thoracoscopic, sternotomy, Myasthenia gravis, thymoma

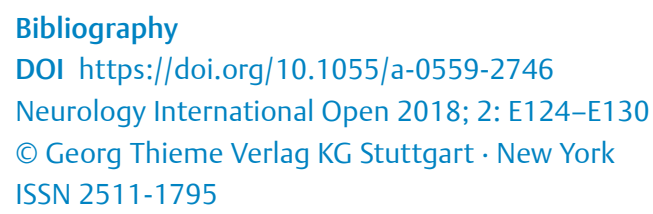

Correspondence

Prof. Dr. med. Jens C. Rückert

Kompetenzzentrum für Thoraxchirurgie (CCM,CVK,CBF)

Chirurgische Klinik Campus Charité Mitte / Campus

Virchow-Klinikum;

Charité - Universitätsmedizin Berlin

Charitéplatz 1

10117 Berlin

Germany

jens-c.rueckert@charite.de

\section{ABSTRACT}

In recent years much progress has been made in the investigation of the pathophysiology, characterizing subgroups, and extension of multimodal treatment of myasthenia gravis (MG). This applies especially to the role of thymectomy (Thx). Thymectomy is always indicated for thymoma-associated myasthenia gravis. Furthermore, based on large cohort studies, during recent decades thymectomy has also become a central part of immune-modulating MG therapy in patients without thymoma. The lack of randomized studies, however, caused a certain persistent reluctance as to the significance of thymectomy. The current MGTX trial has shown the effectiveness of thymectomy. A significant improvement of myasthenic complaints and the reduction of immunosuppressive medication was primarily shown for acquired early-onset MG (EOMG) with complete resection of all thymic tissue. Because the MGTX study only included patients younger than 65 years with generalized MG and positive for acetylcholine-receptor antibodies, at present the significance of Thx for other relevant subgroups as juvenile MG, MG in older patients, ocular MG, as well as seronegative patients is under investigation. Even the prevailing opinion of no benefit of thymectomy for MuSk-positive patients probably needs reevaluation based on ambiguous findings. With respect to surgery, based on the exclusive performance of extended median sternotomy for MG in the MGTX, the value of thoracoscopic modifications for thymectomy as a minimally-invasive alternative is currently under evaluation. For clinical reasons further judgment regarding different minimally-invasive thymectomy techniques compared to the conventional open procedures in the form of randomized comparative studies would be required. Currently, however, an experience-based robotic-assisted thoracoscopic unilateral approach to thymectomy meets all requirements related to surgical, clinical-neurological and patient aspects. Ethical reasons, therefore, will lead to other strategies for comparison of different surgical techniques.

\section{Introduction}

Surgical therapy of myasthenia gravis was the first targeted approach to treating this disease [2]. Long before the autoimmune character of MG was recognized, in 1901 autopsy examinations showed a connection with tumors of the thymus gland [3]. After initially sporadic employment of thymectomy (Thx), there was a latency of a few decades after the historic development of symptomatic therapy with cholinesterase inhibitors in 1934 ("Walker effect"), then surgical therapy was systematically resumed [4-6]. Historical consideration of thymectomy is currently of particular importance, since the present proof of the effectiveness of Thx for the treatment of MG can deductively also be applied to associated issues. The controversy over the surgical treatment of MG has 
changed in content since the principal doubts about the usefulness of thymectomy in the treatment of autoimmune myasthenia gravis have been overcome [1]. Based on the MGTX study, the overview presented here focuses on the current data on the significance of thymectomy for other clinically relevant subgroups, such as juvenile myasthenia gravis (JMG), ocular MG (OMG), late-onset myasthenia (LOMG), thymoma-related MG (including MG with microscopic thymoma), MG without antibodies (so-called seronegative myasthenia) as well as gender-specific differences. The recommended surgical technique is of particular importance to all involved. Is there always a need for a sternotomy, or can a modification of a thoracoscopic procedure be considered as standard? Responses to these issues are provided in light of regional, international and especially experiential differences, since there is currently a rapid change in approach toward a unilateral procedure and robotic-assisted thoracoscopic techniques ( $>$ Table 1, $\triangleright$ Figs. 1,2).

\section{Thymectomy in Different MG Subgroups}

The past 20 years have seen significant advances in the pathophysiological understanding of MG, resulting in a therapeutically-relevant classification into new subgroups [7-9]. The classification principles relevant for thymectomy take into account thymoma association, age of onset and antibody specificity, that is, the issue of whether there are antibodies to the usual AChR antigen (about $80-85 \%$ of patients) or MuSK (muscle-specific receptor tyrosine kinase, about 3-5\%) or LRP4 (low-density lipoprotein receptor-re-

- Table 1 Current techniques

- Sternotomy vs. MIC

- Thoracoscopy vs. thoracoscopy with robotic assistance

- Unilateral vs. bilateral thoracoscopy

- Unilateral thoracoscopy from right or left side

- Thoracoscopy vs. subxiphoidal

- Thoracoscopy vs. transcervical

- Thoracoscopy vs. VATET

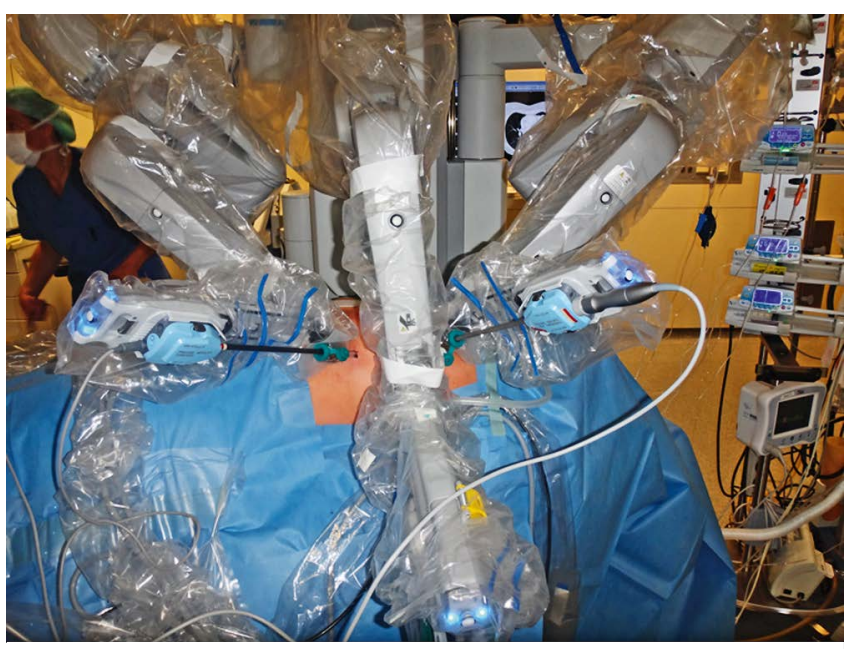

Fig. 1 Positioning. lated protein $4,<1 \%$ ) [8]. About an additional $10-15 \%$ of all patients demonstrate no antibodies; these are designated as "triple seronegative". This is inaccurate insofar as it can be assumed that even seronegative patients have specific disease-causative autoantibodies, which so far have eluded detection and that these are therefore MG patients without identified antibodies.

The MGTX study can now determine with certainty the effectiveness of Thx for the largest group of myasthenia patients with early onset myasthenia gravis (EOMG) and an AChR-antibody-positive course of the disease [1]. A number of thymectomy series have already proven the advantages of the procedure for MG [10-15].
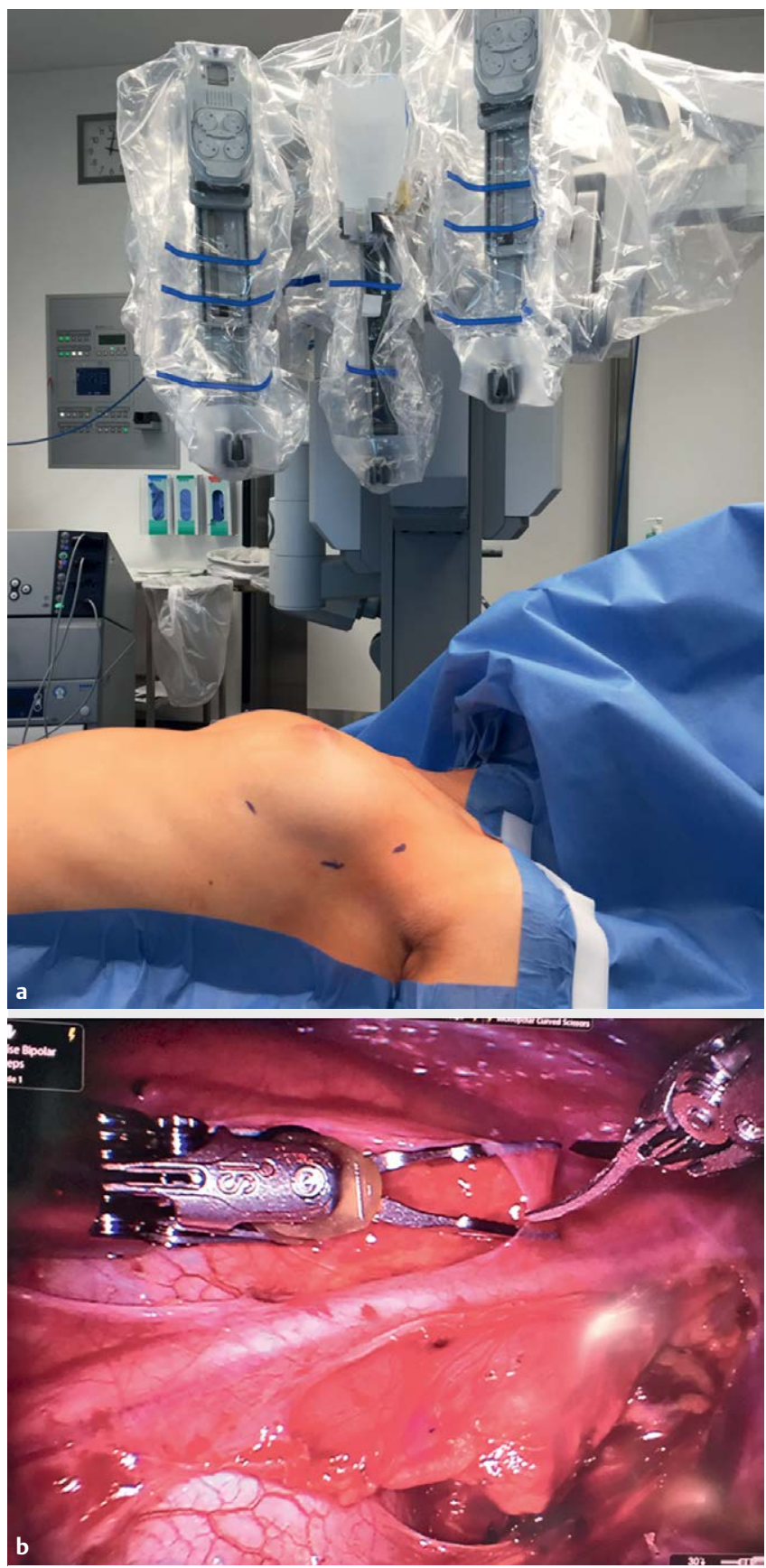

- Fig. 2 a Position of the da Vinci robotic system table cart b Left phrenic nerve after exposure in the operating area 
In 2000 Gronseth and Barohn, based exclusively on comparative studies, showed in a meta-analysis that patients with a generalized progression of EOMG benefited from thymectomy if they underwent surgery early after disease onset ( $<2$ years) [16]. However, since data from a randomized controlled trial was lacking until recently, doubts about the importance of Thx in myasthenia gravis therapy were widespread. Since prior to the MGTX study there were no prospective randomized controlled studies, until recently thymectomy was often ruled out for patients without suspected thymoma despite the above-mentioned meta-analysis and the largely recognized role of the thymus in the pathophysiology of myasthenia gravis [9]. It should be considered that MG is the only autoimmune disease to date in which thymectomy is performed and in principle for which other methods of therapy are available using drug immunosuppression, which were at least tested in (although often not high-quality) studies. A comparison of the data of the meta-analysis with the results of the MGTX study shows a high congruence of the results. Based on the demonstrated high value of Thx in the main group of MG patients [17], it is instructive for the future of myasthenia gravis therapy to consider in more detail the sources and causes of the reluctant or even negative attitude towards thymectomy.

Historically, thymectomy surgery procedures were still very limited until the 1970s. Moreover, effective antibiotics were lacking, the possibilities of intensive care were limited and there were no effective immunosuppressive MG therapies, so that the perioperative morbidity of thymectomy was comparatively high in the early days of the procedure. In the first systematic series published by Blalock, the perioperative mortality of 20 patients was $25 \%$ [5]. He had already recognized the need for an extensive or complete thymectomy [18], since at that time neither the significance of any existing ectopic mediastinal thymic tissue nor the autoimmune genesis of myasthenia gravis was known. Long before minimally-invasive thoracoscopy revolutionized the treatment of myasthenia gravis, the return of the original transcervical approach via jugular transverse incision around 1970 started the debate on how thymectomy should be performed [12,18-20]. Until the introduction of the thoracoscopic surgical technique, the central debate between advocates of the cervical or transsternal surgical technique had not been conclusively determined with regard to the analysis of the respective results. Therefore in 1997 Jaretzki established a myasthenia gravis task force to statistically evaluate the results of surgical therapy of the disease [21]. This approach led to the systematic investigation of the importance of thymectomy in MG therapy; a component of this study was the above-mentioned meta-analysis by Gronseth and Barohn which formed the basis of the MGTX study [16]. Consequently, the inclusion criteria focused on the core group of early-onset myasthenia gravis patients. As a result only limited conclusions are possible for the other typical MG subgroups.

Consideration of the status quo based on the meta-analysis of 2000 and the MGTX study provide the following picture. The MGTX study demonstrated that cases of generalized myasthenia gravis with positive detection of AchR antibodies, disease onset age below 65 years and a disease history of less than 5 years (according to the 2000 meta-analysis < 2 years) would benefit from thymectomy. Based on the inclusion criteria of the MGTX study, patients younger than 18 years of age or older than 65 as well as patients without verified AChR antibodies were not studied. As a result, there are uncertainties regarding these groups of patients which should be addressed here in the sense of the indication and recommended surgical technique based on the available evidence.

\section{Thymectomy in Juvenile Myasthenia Gravis}

Thymectomy for children and adolescents poses particular technical, ethical and medical challenges. The earlier concept, that due to the possible role of the thymus in the maturation of the immune system, thymectomy had to be delayed until puberty, is countered by the fact that the time interval between the first manifestation of MG and the thymectomy inversely correlates with the therapeutic effect. Based on current neonatal cardiosurgical data which regularly "sacrifices" thymus tissue, there is no evidence of immunological disadvantages of thymectomy in early childhood [22]. No negative effects have been known from the comparatively few cases of juvenile myasthenia gravis. However, risk-benefit analysis must take into account that drug immunosuppression can lead to growth retardation; thymectomy can reduce this risk. Our range of experience includes thymectomy in children from the age of 4 years; internationally, success has even been reported in individual cases from the age of 1.5 years. The requirements for an appropriate surgical procedure are best met by a minimally-invasive surgical technique, in particular unilateral robotic-assisted thymectomy. A recent systematic review supports the use of Thx in children with AChR-ab-positive myasthenia gravis [23]. Based on retrospective data from a total of 1131 patients (43\% with thymectomy) published in the years 2000 to 2016, a significant advantage of Thx-treated patients was found in comparison to those exclusively receiving conservative therapy [24]. Of the 488 thymectomy patients, however, only 70 had undergone thoracoscopic surgery, and only a very small proportion had been operated on with robotic assistance [24]. In our recently published case series of 18 patients with juvenile-onset MG who underwent exclusively robotic-assisted surgery, we demonstrated the superiority of this technique, which is reflected in the very good long-term results combined with the absence of related side effects [25].

\section{Thymectomy in Older Patients}

The second age peak of the incidence of MG begins at the age of 40 and is defined as a late manifestation (late-onset myasthenia gravis, LOMG), whereas the age limit of 40-65 years can be highly variable [26]. The increasing incidence and prevalence of LOMG is potentially due to the growing average age of the population, improved diagnostic capabilities, and possibly also increasing polypharmacy in older age with drugs, some of which are suspected of being MG triggers [7]. Annually, more than $60 \%$ of all newly-diagnosed myasthenia gravis have LOMG [7]. The are various reasons to treat LOMG patients with thymectomy, although the age limit for this surgery has steadily increased over the last two decades and is now often given as 60 years. One of the main reasons is natural thymic involution, i. e. atrophy of the thymus with increasing age. On the other hand, the pathophysiological assumption is that fol- 
licular hyperplasia ("thymitis") is an etiological correlate of MG. There are no systematic studies with larger case numbers, since due to the above-mentioned age limit, older patients are not operated on; however some of these patients also have follicular hyperplasia, albeit less frequently than younger ones. Another reason was the sometimes high morbidity of sternotomy in the past among the often multimorbid elderly patients. In addition, the generally lower remaining life expectancy of older patients is used as an argument. This contrasts with data showing that even older patients with generalized MG can benefit from thymectomy [27]. Based on current data and evidence of the occurrence of follicular hyperplasia among older patients, European guidelines recommend thymectomy also in cases of late-onset MG [28]. Furthermore, in the MGTX study, which due to slow recruitment, extended the inclusion criteria to involve older patients, the results for the late-onset myasthenia gravis group were similar to the early-onset group. A minimally-invasive thymectomy technique, especially using robotic assistance, is highly suited to operate without delay or risk of destabilizing the MG due to interruption of immunosuppressive therapy.

\section{Thymectomy for Ocular Myasthenia Gravis}

The issue of whether a patient with OMG should be offered a thymectomy is still under discussion. First, the therapeutic goal must be defined. A basic aim may be to reduce the symptoms as in the generalized form and/or to reduce or discontinue drug immunosuppressive therapy. Since the ocular form of myasthenia gravis is generally regarded as not as disabling as the generalized form, this therapeutic goal is frequently rejected due to the lack of random clinical trial evidence. It should be pointed out that many patients with the ocular form of MG suffer a high level of psychological stress and must as a rule be treated with immunosuppressive drugs or have to undergo ophthalmosurgical therapy. However, it has long been known that approx. $50-70 \%$ of ocular cases become generalized. In these cases, there is an $80-90 \%$ probability of generalization within the first 2 to 3 years [29]. In addition, purely ocular forms are rare in the long term. This results in a second possible therapy goal for the thymectomy, the prevention of generalization. The surgery is more effective the sooner it is performed after disease onset; thus it does not make sense to wait until it becomes generalized. There are no widely-accepted markers that are predictive of generalization. However, long-term purely ocular progressions are seronegative in approx. $50 \%$ of cases or have very low AChR-antibody titers (see below for the importance of so-called clustered AchR antibodies [30].

Since Keynes' comprehensive early analysis, most researchers have found a significant advantage in performing thymectomy early, making waiting for generalization at the onset of ocular MG risky [10,31-34]. A recent meta-analysis based on 26 publications has analyzed the course of 684 patients with ocular MG and thymectomy [35]. The pooled rate of completely stable full remission was comparably high at $51 \%$. The results of thymectomy were better in European and American patients than in Asian patients, and in children better than in adults [35]. These data are an indica- tion that thymectomy can also effectively improve ocular MG. Further systematic data analysis is needed in order to better estimate the therapeutic effects on ocular symptoms and generalization. However, the extent to which multi-center, randomized, controlled clinical trials on the effects of thymectomy, especially in this subgroup, is necessary before this therapy should be recommended for initial onset of ocular MG, is a controversial topic. In this case, approximately $30 \%$ of generalized AChR-ab-positive MG patients (with ocular symptoms) would be deprived of effective therapy at the optimal juncture (as early as possible after disease onset) according to the MGTX study.

\section{Significance of Antibody Status in the Decision for Thymectomy}

The thymectomy data are largely based on AChR-ab-positive myasthenia gravis patients. The limited body of data relating to the effectiveness of thymectomy in MuSK-ab-positive MG are contradictory; typical thymic pathologies (thymoma, thymic hyperplasia) have been observed only in exceptional cases [26, 36, 37]. Interestingly, thymic changes are apparent, the significance of which is still unclear [37]. Therefore, based on this data, the guidelines do not recommend thymectomy for MuSK-ab-positive patients. However, in our view, imaging should be used to find thymic pathologies for this type of MG, and thymectomy performed in cases of relevant suspicion. In addition, thymectomy should be performed for individual cases of severe treatment-refractory disease.

There are no meaningful data available regarding the value of thymectomy in cases of LRP4-ab-positive MG which is very rare in Germany (<1\%) [38].

Data regarding thymectomy for seronegative patients date mainly from the time when new autoantibodies (especially against MuSK) were not available. In addition, not a small proportion of seronegative patients (especially ocularly affected) probably have positive evidence of so-called cluster AChR antibodies, which are only detectable in a commercially unavailable cell-based assay [39]. Among seronegative patients, the proportion of follicular hyperplasia is about $30 \%$ and thus less common compared to AChR-ab-positive EOMG (about 70\%) [36]; therefore thymectomy should also be considered for such patients. Systematic data collection (e. g., in registries) and prospective studies to examine the importance of thymectomy for these subgroups are needed.

\section{Thymectomy for Thymoma and Myasthenia Gravis}

Thymoma or the suspicion of thymoma are absolute indications for surgery. With a diagnosis of MG, (contrast) CT or MRI-based imaging is required for this constellation, although small thymomas, microthymomas, and so-called microscopic thymomas can inherently elude these diagnostic methods [30]. Despite the successful description of minimally-invasive thymectomy techniques, sternotomy was still considered the sole safe method in some centers [40]. The initial concern about possible higher recurrence rates due to injury of the thymic capsule and/or non-radical thymectomy and 
lower rates of improvement of MG has not been confirmed in technically perfect thoracoscopic thymectomies [41-45]. Nowadays thymectomy can be performed on Masaoka-Koga stages I and II using minimally-invasive techniques [41-43]. The size of the thymoma is not an absolute criterion for an adequate surgical technique. Consistent with the experience gained at the Charité in Berlin after 115 robotic-assisted thymectomies, groups in Europe, Asia and the USA have also partially demonstrated using propensity-matching design that a robotic-assisted thoracoscopic surgical technique can be adequate for larger thymomas [44-46]. Subxiphoid fourth trocar access or subxiphoidal extirpation is recommended for larger thymomas.

\section{Thymectomy in Adipose Patients with Myasthenia Gravis}

The MGTX study was able to dispel the remaining doubts regarding the indication for thymectomy in the main group of MG patients. The basic risk-benefit assessment of therapies mainly concerns surgical procedures. This applies in particular to elective thymectomy for MG; the perioperative complication rate was always a central component of the discussion in addition to the indication for the surgical procedure ( $\triangleright$ Fig. 1$)$. It has been assumed that only an open surgical technique (sternotomy) can be sufficiently radical in the complete removal of the thymus gland. However, this is not the case, minimally invasive procedures are now at least equivalent in terms of thoroughness. On the other hand, an open surgical technique is more likely to cause complications and interfere with the underlying disease and comorbidities. For example, a recent study of patients with obesity (BMI > 23.3) shows a significantly higher rate of complications, especially respiratory failure and extended hospitalization [47]. The technical possibilities of robotic assistance offer the likelihood of an extremely low-complication complete minimally invasive thymectomy even for MG patients with a higher BMI.

\section{How Should the Thymectomy be performed?}

Twenty years ago "Aktuelle Neurologie” (English: Current Neurology) in a special issue devoted to myasthenia gravis published an article introducing minimally-invasive thymectomy [48] in which the thoracoscopic thymectomy technique was described and established in detail [49-51]. Five years earlier, thoracoscopic thymectomy had been performed for the first time, followed by a small series of thymectomies $[50,52,53]$. Since then, the surgical technique has evolved enormously, and issues regarding the benefits of individual procedures have become the subject of research ( $\triangleright$ Fig. 1).

The "MG Task Force" previously mentioned, was founded by Jaretzki in 1997; the meta-analysis by Gronseth and Barohn (2000) provided convincing data for the effectiveness of thymectomy in the treatment of MG [21]; the MGTX study was intended to provide final evidence [1]. It should be noted that there was a 15-year interval between the planning of the study and its successful conclu-

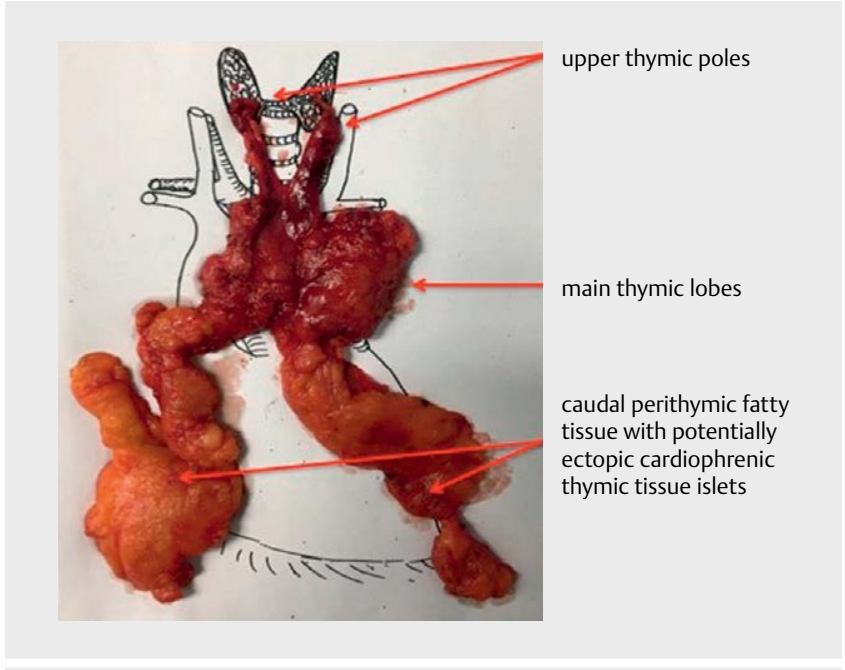

Fig. 3 Preparation after resection.

sion. This has to do with the surgical procedure used, among other things [1]. Extended median sternotomy was uniformly established as the surgical technique for the MGTX study. Based on this study, it might be concluded that selection of this access method was mandatory, since an advantage was shown only for this procedure. Do minimally-invasive thymectomy procedures have the same value? The body of data 20 years after the development of minimally-invasive thymectomy surgical techniques support this. Thus, the efficacy of sternotomy and unilateral simple thoracoscopy was comparable [14]. A recent retrospective study using propensity analysis sees advantages compared to sternotomy with respect to improving myasthenic symptoms after extended thoracoscopic technique with supplementary neck and subxiphoidal access (VATET technique, > Table 1) [54]. The robotic-assisted thoracoscopic technique currently provides the greatest advantages for a mixed patient population of MG and/or thymoma patients since technical advantages and systematic familiarization allow optimal radical thymus removal even under difficult individual anatomical conditions ( $\triangleright$ Figs. 1-4) [41, 55-58]. Robotic assistance resulted in a significantly higher rate of complete remission of MG after minimally-invasive thoracoscopic thymectomy [59]. The surprisingly clear positive results of the MGTX study have further accelerated the trend towards the introduction of minimally-invasive thymectomy techniques. However, seen globally, there are differences. One example of this is the greater importance of the discussion of sternotomy vs. thoracoscopy in Europe, whereas in Asia the focus is almost exclusively on optimal minimally-invasive surgical technique. - Table 1 provides an overview of the current issues in this regard. There are clear arguments for performing a left-side unilateral robotic-assisted thymectomy with 3 trocars ( $\triangleright$ Figs. 1-4). Japanese colleagues have described a bilateral technique, since only in this way were they able to perform radical thymic tissue removal on the contralateral diaphragm [60]. A dedicated technique underlies the left-side 3-trocar access we have introduced [61, 62] which can be taught in a systematic staged learning program. The missing difference between left- and right-side thymectomy is due to the somewhat low quality of available literature on this topic 


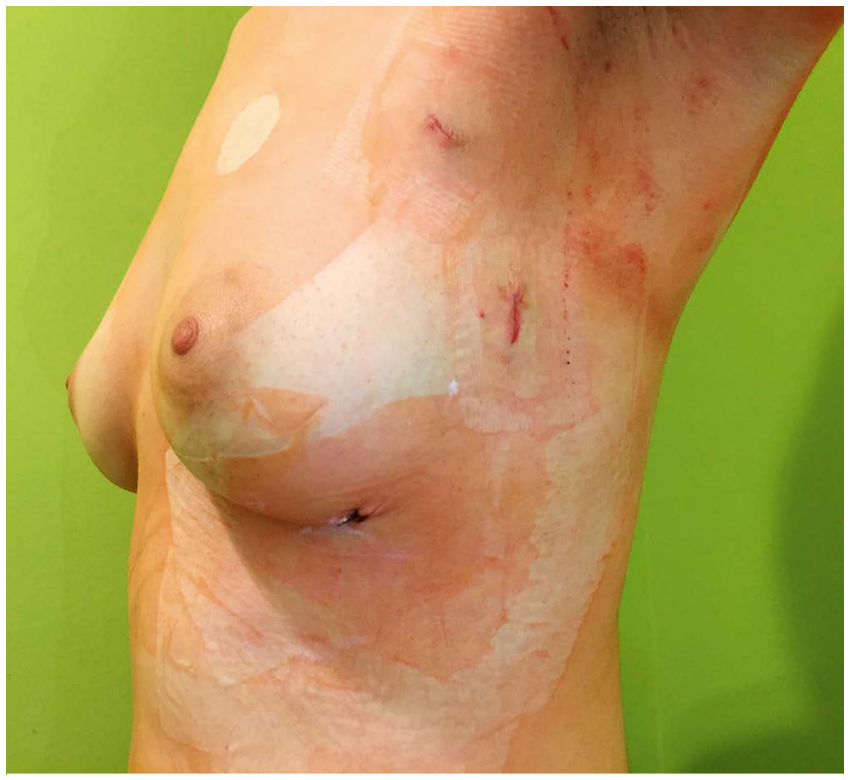

Fig. 4 Incisions on day 1 after surgery.

[63]. The anatomical variability of the left part of the thymus is the main reason why a change to the left-sided approach was made even in the case of primarily right-sided surgery (Cerfolio, personal report) [41]. In this context it is crucial to perform a radical thymectomy in any case ( $>$ Fig. 3 ). All critics of minimally-invasive thymectomy techniques have the legitimate concern that this principle may be risky, given a lack of experience, aggravating anatomical variations, increased BMI or complex thymomas.

\section{Summary}

In the case of a thymoma, thymectomy is not only essential for the treatment of myasthenia gravis, but also to prevent vascular, cardiac, pulmonary or nerve damage caused by local tumor growth. Even if there is no suspicion of thymoma, AChR-ab-positive MG patients younger than 66 years should be offered thymectomy early after disease onset (Evidence level Ib) Thymectomy may also be considered for AChR-ab-positive patients older than 65 years with seronegative and ocular myasthenia gravis. Currently thymectomy is not recommended for the MuSK-ab-positive MG subgroup. The value of thymectomy should be investigated in additional clinical studies of such subgroups. Surgical technique should be oriented toward the completeness of thymic tissue removal, in which case ectopic thymus tissue may also have to be excised ( $\vee$ Fig. 3 ). This goal can be achieved with sufficient experience with thoracoscopic surgical techniques. A minimally-invasive radical complete thymectomy can be particularly obtained with robotic assistance ( Fig. 4).

\section{Conflict of Interest}

The authors declare no conflict of interest.

\section{References}

[1] Wolfe GI, Kaminski HJ, Aban IB et al. Randomized trial of thymectomy inmyasthenia gravis. N Engl J Med 2016; 375: 511-522

[2] Schumacher ED, Roth J. Thymektomie bei einem Fall von Morbus Basedowi mit Myasthenie. Mitt Grenzgeb Med Chir 1912; 25: 746 $-765$

[3] Laqueur L, Weigert C. Beiträge zur Lehre von der Erb'schen Krankheit. Neurol Zbl 1901; 20: 4

[4] Blalock A, Mason MF, Morgan HJ et al. Myasthenia gravis and tumors of the thymic region: Report of a case in which the tumor was removed. Ann Surg 1939; 110: 544 - 561

[5] Blalock A. Thymectomy in the treatment of myasthenia: Report of 20 cases. J Thorac Surg 1944; 13: 316 - 336

[6] Blalock A, McGehee $H$, Ford FR et al. The treatment of myasthenia gravis (by) removal of the thymus gland: Preliminary report. JAMA 1941; 117: 1529 - 1533

[7] Vincent A, Palace J, Hilton-Jones D. Myasthenia gravis. Lancet 2001; 357: 2122 - 2128

[8] Gilhus NE, Verschuuren J]. Myasthenia gravis: Subgroup classification and therapeutic strategies. Lancet Neurol 2015; 14: 1023 - 1036

[9] Sieb JP. Myasthenia gravis: An update for the clinician. Clin Exp Immunol 2013; 175: 10

[10] Keynes G. The results of thymectomy in myasthenia gravis. Br Med ] 1949; 2: $611-616$

[11] Shrager JB, Nathan D, Brinster C] et al. Outcomes after 151 extended transcervical thymectomies for myasthenia gravis. Ann Thorac Surg 2006; 82: 1863 - 1869

[12] Jaretzki A 3rd, Penn AS, Younger DS et al. "Maximal” thymectomy for myasthenia gravis. Results. J Thorac Cardiovasc Surg 1988; 95: 747 $-757$

[13] Jaretzki A 3rd. Video-assisted thoracoscopic extended thymectomy and extended transsternal thymectomy in non-thymomatous myasthenia gravis patients. J Neurol Sci 2004; 217: 233-234 Author reply $235-236$

[14] Tomulescu V, Sgarbura O, Stanescu C et al. Ten-year results of thoracoscopic unilateral extended thymectomy performed in nonthymomatous myasthenia gravis. Ann Surg 2011; 254: 761-765 Discussion 765-766

[15] Bulkley GB, Bass KN, Stephenson GR et al. Extended cervicomediastinal thymectomy in the integrated management of myasthenia gravis. Ann Surg 1997; 226: 324-334 Discussion 334-325

[16] Gronseth GS, Barohn RJ. Practice parameter: thymectomy for autoimmune myasthenia gravis (an evidence-based review): Report of the Quality Standards Subcommittee of the American Academy of Neurology. Neurology 2000; 55: 7-15

[17] Sieb JP. Myasthenia gravis: An update for the clinician. Clin Exp Immunol 2014; 175: 408 - 418

[18] Sonett JR, Magee M], Gorenstein L. Thymectomy and myasthenia gravis: A history of surgical passion and scientific excellence. J Thorac Cardiovasc Surg 2017; 154: 306 - 309

[19] Kark AE, Kirschner PA. Total thymectomy by the transcervical approach. Br J Surg 1971; 58: 321 - 326

[20] Cooper JD, Al-jilaihawa AN, Pearson FG et al. An improved technique to facilitate transcervical thymectomy for myasthenia gravis. Ann Thorac Surg 1988; 45: 242 - 247

[21] Jaretzki A 3rd. Thymectomy for myasthenia gravis: Analysis of controversies regarding technique and results. Neurology 1997; 48 (Suppl. 05):52S - 63S. doi: https://doi.org/10.1212/WNL.48Suppl_ 5.525 
[22] Hermes HM, Cohen GA, Mehrotra AK et al. Association of thymectomy with infection following congenital heart surgery. World J Pediatr Congenit Heart Surg 2011; 2: 351 - 358

[23] Gui M, Luo X, Lin J et al. Long-term outcome of 424 childhood-onset myasthenia gravis patients. J Neurol 2015; 262: 823 - 830

[24] Madenci AL, Li GZ, Weil BR et al. The role of thymectomy in the treatment of juvenile myasthenia gravis: A systematic review. Pediatr Surg Int 2017; 33: 683 - 694

[25] Della Marina A, Kolbel H, Mullers M et al. Outcome after robotic-assisted thymectomy in children and adolescents with acetylcholine receptor antibody-positive juvenile myasthenia gravis. Neuropediatrics 2017; 48: 315 - 322

[26] Binks S, Vincent A, Palace J. Myasthenia gravis: A clinical-immunological update. J Neurol 2016; 263: 826 - 834

[27] Kawaguchi N, Kuwabara S, Nemoto $Y$ et al. Effects of thymectomy on late-onset myasthenia gravis without thymoma. Clin Neurol Neurosurg 2007; 109: 858 - 861

[28] Skeie GO, Apostolski S, Evoli A et al. Guidelines for treatment of autoimmune neuromuscular transmission disorders. Eur J Neurol 2010; 17: 893 - 902

[29] Grob D, Brunner N, Namba T et al. Lifetime course of myasthenia gravis. Muscle Nerve 2008; 37: 141-149

[30] Schumm F, Dichgans ]. [Clinical evaluation system (score) of ocular symptoms in myasthenia gravis]. Nervenarzt 1985; 56: 186 - 187

[31] Tansel T, Onursal E, Barlas S et al. Results of surgical treatment for nonthymomatous myasthenia gravis. Surg Today 2003; 33: 666 - 670

[32] Luo LQ, Zhang JZ, Cui HN. Clinical analysis of thymectomy for juvenile with myasthenia gravis. China J Mod Med 2008; $15: 2$

[33] Xie SP, Huang J. A clinical assessment of thymectomy for the treatment of ocular myasthenia gravis in children. J Clin Surg 2010; 18: 2

[34] Lui Z, Feng $\mathrm{H}$, Yeung SC et al. Extended transsternal thymectomy for the treatment of ocular myasthenia gravis. Ann Thorac Surg 2011; 92: 7

[35] Zhu K, Li ], Huang X et al. Thymectomy is a beneficial therapy for patients with non-thymomatous ocular myasthenia gravis: A systematic review and meta-analysis. Neurol Sci 2017; 38: 1753 - 1760

[36] Lauriola L, Ranelletti F, Maggiano N et al. Thymus changes in anti-MuSK-positive and -negative myasthenia gravis. Neurology 2005; 64: $536-538$

[37] Leite MI, Strobel P, Jones M et al. Fewer thymic changes in MuSK antibody-positive than in MuSK antibody-negative MG. Ann Neurol 2005; 57: 444-448

[38] Ponseti JM, Caritg N, Gamez J et al. A comparison of long-term postthymectomy outcome of anti-AChR-positive, anti-AChR-negative and anti-MuSK-positive patients with non-thymomatous myasthenia gravis. Expert Opin Biol Ther 2009; 9: 1 - 8

[39] Gilhus NE, Skeie GO, Romi F et al. Myasthenia gravis - autoantibody characteristics and their implications for therapy. Nat Rev Neurol 2016; 12: $259-268$

[40] Wright CD. Management of thymomas. Crit Rev Oncol Hematol 2008; 65: $109-120$

[41] Marulli G, Rea F, Melfi F et al. Robot-aided thoracoscopic thymectomy for early-stage thymoma: A multicenter European study. J Thorac Cardiovasc Surg 2012; 144: 1125 - 1130

[42] Marulli G, Maessen J, Melfi F et al. Multi-institutional European experience of robotic thymectomy for thymoma. Ann Cardiothorac Surg 2016; 5: 18 - 25

[43] Fukuhara M, Higuchi M, Owada Y et al. Clinical and pathological aspects of microscopic thymoma with myasthenia gravis and review of published reports. J Thorac Dis 2017; 9: 1592-1597
[44] Keijzers M, Dingemans AM, Blaauwgeers H et al. 8 years' experience with robotic thymectomy for thymomas. Surg Endosc 2014; 28: 1202 $-1208$

[45] Kang CH, Hwang Y, Lee $\mathrm{H}$ J et al. Robotic thymectomy in anterior mediastinal mass: Propensity score matching study with transsternal thymectomy. Ann Thorac Surg 2016; 102: 895-901

[46] Kneuertz PJ, Kamel MK, Stiles BM et al. Robotic thymectomy is feasible for large thymomas: a propensity-matched comparison. Ann Thorac Surg 2017; 104: 1673-1678

[47] Liu XD, Shao MR, Sun L et al. Influence of body mass index on postoperative complications after thymectomy in myasthenia gravis patients. 2017; www.impactjournals.com/oncotarget/

[48] Gellert K. Die thorakoskopische Thymektomie. Akt Neurol 1998; 25: $570-572$

[49] Ruckert JC, Gellert K, Einhaupl K et al. [Thoracoscopic thymectomy for treatment of myasthenia gravis]. Zentralbl Chir 1998; 123: 506- 511

[50] Mineo TC, Pompeo E, Ambrogi V et al. Adjuvant pneumomediastinum in thoracoscopic thymectomy for myasthenia gravis. Ann Thorac Surg 1996; 62: $1210-1212$

[51] Ruckert JC, Gellert K, Muller JM. Operative technique for thoracoscopic thymectomy. Surg Endosc 1999; 13: 943 - 946

[52] Landreneau RJ, Dowling RD, Castillo WM et al. Thoracoscopic resection of an anterior mediastinal tumor. Ann Thorac Surg 1992; 54: 142 144

[53] Mack M], Landreneau RJ, Yim AP et al. Results of video-assisted thymectomy in patients with myasthenia gravis. J Thorac Cardiovasc Surg 1996; 112: 1352-1359 Discussion 1359-1360

[54] Brenna G, Antozzi C, Montomoli C et al. A propensity score analysis for comparison of T-3b and VATET in myasthenia gravis. Neurology 2017; 89: $189-195$

[55] Ruckert JC, Ismail M, Badakhshi $\mathrm{H}$ et al. [Thymectomy in myasthenia and/or thymoma]. Zentralbl Chir 2014; 139: 121-132 quiz 133-124

[56] Ismail M, Swierzy M, Ruckert JC. State of the art of robotic thymectomy. World J Surg 2013; 37: 2740-2746

[57] Ismail M, Swierzy M, Ruckert RI et al. Robotic thymectomy for myasthenia gravis. Thorac Surg Clin 2014; 24: 189-195 vi-vii

[58] Marulli G, Schiavon M, Perissinotto E et al. Surgical and neurologic outcomes after robotic thymectomy in 100 consecutive patients with myasthenia gravis. J Thorac Cardiovasc Surg 2013; 145: 730-735 discussion 735-736

[59] Ruckert JC, Swierzy M, Ismail M. Comparison of robotic and nonrobotic thoracoscopic thymectomy: A cohort study. J Thorac Cardiovasc Surg $2011 ; 141: 673-677$

[60] Kawaguchi K, Fukui T, Nakamura S et al. A bilateral approach to extended thymectomy using the da Vinci Surgical System for patients with myasthenia gravis. Surg Today 2018; 48: 195- 199

[61] Rückert JC, Swierzy M, Rückert RI et al. Thymectomy (VATS, da Vinci). In: Dienemann HC, Hoffmann H, Detterbeck FC. (eds). Chest Surgery: Springer Surgery Atlas Series. Springer; 2014

[62] Ismail M, Swierzy M, Rückert RI et al. Robotic thymectomy for myasthenia gravis. Thorac Surg Clin 2014; 24: 189-195

[63] Elsayed HH, Gamal M, Raslan S et al. Video-assisted thoracoscopic thymectomy for non-thymomatous myasthenia gravis: a right-sided or left-sided approach? Interact Cardiovasc Thorac Surg 2017; 25: 651-653 